\title{
Design and Experiment of NIR Wheat Quality Quick Detection System*
}

\author{
Lingling Liu ${ }^{* *}$, Bo Zhao ${ }^{* * *}$, Yinqiao Zhang, and Xiaochao Zhang \\ National Key Laboratory of Soil-Plant-Machine System, \\ Chinese Academy of Agricultural Mechanization Sciences, Beijing, China, 10083 \\ whyispasina.com, zhaoboshi@126.com
}

\begin{abstract}
In this paper, NIR wheat quality quick detection system (NIR-WQDS) was developed on the base of grating technology with a scanning range of 900-1700nm.46 wheat samples were analyzed to compare performance of NIR-WQDS and MPA FT-NIR spectroscopy (MPA). Experiment results of NIR-WQDS show that the coefficient of determination $\mathrm{R}^{2}$ is $94.44 \%$, root mean square error of cross validation RMSECV is 0.3460 , ratio of performance to standard deviate RPD is 4.240, and root mean square error of prediction RMSEP is 0.2430 , respectively. The model of MPA spectroscopy gave an $R^{2}$ of $95.95 \%$, a RMSECV of 0.2950 , a RPD of 4.970, and an RMSEP of 0.2270 respectively. It is found that spectra of NIR-WQDS have a roughly same changing trend. NIR-WQDS can detect wheat quality with good accuracy, repeatability and stability, which is similar to MPA. The results show that NIR-WQDS can work steadily with a good performance.
\end{abstract}

Keywords: NIR spectroscopy, Wheat quality, Quick detection system, Quantitative analysis, Predictive performance.

\section{Introduction}

Near infrared spectroscopy (NIRS) technique is characterized by simple operation, short period and environmental friendliness. Compared with Kjeldahl nitrogen method, NIRS technique can detect protein of wheat quickly and nondestructively, which is meaningful for wheat quality inspection, processing and breeding. NIRS technique is constituted by NIR instrument, chemometrics software and calibration model [1] As NIRS instrument is becoming miniature, professional, steady and excellent, domestic and foreign famous companies have developed kinds of the special purpose instruments focused on inspecting grain, feed, soil and oil [2]. Domestic researchers have already gained some achievements in wheat spectroscopy research, but our research is still far

\footnotetext{
* This work was supported by national science and technology support program for the 12th five-year plan (2011BAD20B06), and national basic research program of China (2010CB735707).

** Major in automatic measuring and control technology.

**** Corresponding author.
}

D. Li and Y. Chen (Eds.): CCTA 2012, Part II, IFIP AICT 393, pp. 135-141, 2013.

(C) IFIP International Federation for Information Processing 2013 
lower than international level [3]. In order to analysis wheat quality professionally and quickly, NIR-WQDS was developed on grating technology and it works in the 900-1700nm region. In this paper 46 wheat samples were detected by MPA spectroscopy and NIR-WQDS to test the performance of NIR-WQDS.

\section{Development of System}

NIR-WQDS includes optical system with fixed grating, CCD drive and signal acquisition system, CCD temperature control system, light source control system, sample rotator control system, and computer software system. NIR absorption spectra are recorded in the $900-1700 \mathrm{~nm}\left(11111.1-5887.8 \mathrm{~cm}^{-1}\right)$ range. CCD is controlled to work at $0^{\circ} \mathrm{C}$ and its temperature controlling precision is $\pm 0.1^{\circ} \mathrm{C}$. The light intensity accuracy can be controlled within $0.02 \%$ and the SNR is 4000:1. The system structure of NIR-WQDS is shown in figure 1.

Following are working principles of NIR-WQDS spectroscopy:

(1) Light source control system is based on current negative feedback. It controls light intensity of source and provides stable near infrared light to the whole instrument.

(2) After light concentrated and collimated, it penetrates wheat sample directly in a sample rotator. Then, diffuse transition light is obtained.

(3) In optical system, diffuse transition light is concentrated and collimated. Then, light is projected onto fixed optical grating and a continuum spectrum is produced.

(4) Cooled CCD detector is driven to sample the spectrum signals and converts it to electric signals. The working temperature of CCD detector is cooled and balanced by CCD temperature control system.

(5) Signal sampling system performs fast, real time data gathering and communicates with upper computer. Then software system in the upper computer processes signals acquired.
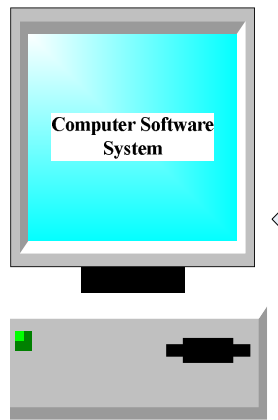

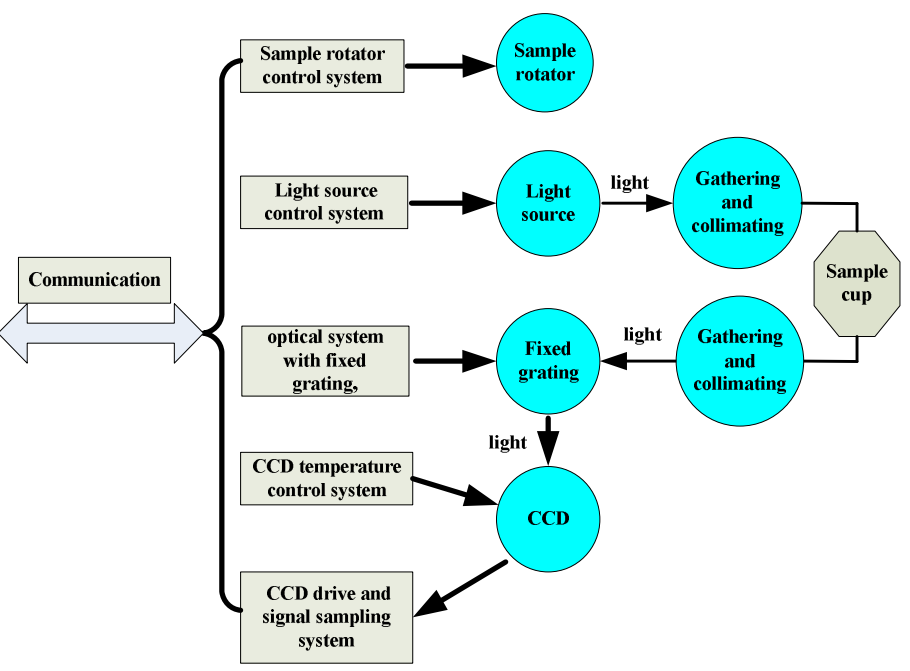

Fig. 1. Structure of NIR-WQDS 
The main characteristics of NIR-WQDS are as follows:

(1) To collect more hydrogen information in wheat groups, spectrum wavelength ranges from $900 \mathrm{~nm}$ to $1700 \mathrm{~nm}$.

(2) To eliminate influence caused by temperature shift and ensure high measurement precision, CCD temperature control system was designed to ensure CCD's working temperature low and steady.

(3) Light intensity control system was designed to ensure stable light with accuracy $0.02 \%$, which reduces error from light intensity shift.

(4) Wheat sample is heterogeneous and inhomogeneous; therefore sample rotator was designed to eliminate error by increasing number of scans [1].

\section{Materials and Methods}

\subsection{Experiment Materials}

46 wheat kernel samples were brought from Heilongjiang academy of agricultural sciences. After milling, the samples were sealed in plastic bags. Protein standard values of samples were measured by national standard method, and it varies representatively and widely from $9.830 \%$ to $16.94 \%$ with a $14.18 \%$ average.

MPA was selected as the target analyzer, which is a fourier transform infrared spectrometer produced by Bruker Optics Company. It has a scanning range of 12800$3600 \mathrm{~cm}^{-1}$. The NIR spectra of 46 wheat powder samples were collected using quartz sample cup on sample rotator in this experiment. The main parameters and setting of two spectrometers are shown in table 1.

Table 1. Main parameters and setting of two instruments

\begin{tabular}{ccccc}
\hline Spectrometer & Spectra Range $\left(\mathrm{cm}^{-1}\right)$ & Sample Cup & Detector & Modeling software \\
\hline NIR-WQDS & $11111.1-5887.8$ & quartz & InGaAs & OPUS6.5 \\
MPA & $12800-3600$ & quartz & PbS & OPUS6.5 \\
\hline
\end{tabular}

\subsection{Experiment Methods}

The spectra were acquired with MPA and NIR-WQDS at room temperature $\left(20 \sim 25^{\circ} \mathrm{C}\right)$ respectively. In order to decreasing operation differences, both instruments were started at the same time for about half an hour. After completing all measurements, the obtained data was processed into absorbance value by using MATLAB 7.0 software. Wheat NIR spectral data was finally analyzed using OPUS 6.5 software. In OPUS6.5, partial least squares (PLS) regression was used to build calibration models. 


\section{$4 \quad$ Results and Analysis}

\subsection{Wheat NIR Spectra}

46 spectra collected by both instruments were saved as OPUS and showed in OPUS6.5 software, see figure 2. Because of different component contents, there is no absolutely general overlapping among all spectra. All spectra have a good identity without any anomaly. Ranging from $11111.1 \mathrm{~cm}^{-1}$ to $5887.8 \mathrm{~cm}^{-1}$, each of NIR spectra has a roughly same changing trend and intendancy with a little difference in peak shape and peak position. In both pictures, wheat protein absorption band with peaks appears at about $10990 \mathrm{~cm}^{-1}, 9804 \mathrm{~cm}^{-1}, 6623 \mathrm{~cm}^{-1}$ and $5051 \mathrm{~cm}^{-1}$. It shows that NIR-WQDS has a stable performance and a good reproductively of sampling wheat NIR spectra.

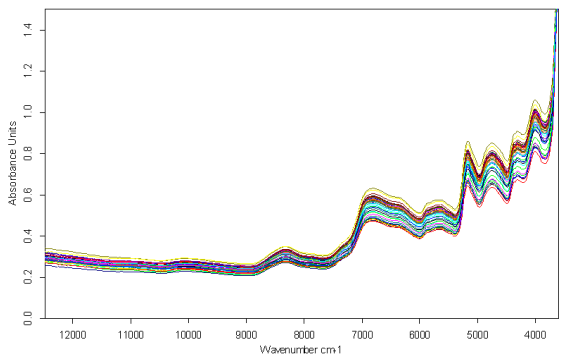

(a)

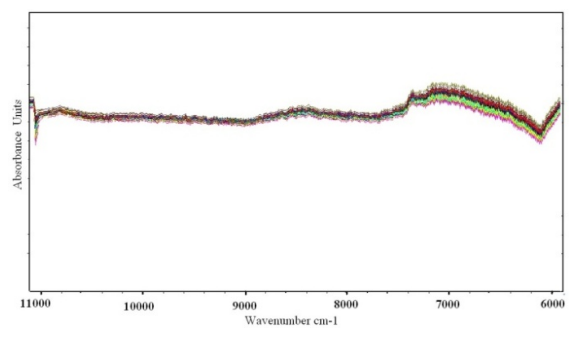

(b)

Fig. 2. 46 wheat sample spectra: (a) MPA (b) NIR wheat quality quick detection system

Comparing with the MPA scans, NIR-WQDS scans are thorny and not smooth with spike-waves at local wavelength and extreme ends, which shows obvious noise and low SNR. To improve the quality of spectra, we can increase scans and improve precision of light control system, and decrease affection caused by CCD dark current.

\subsection{Peprocessing and Modeling Methods}

In this paper, PLS were selected for building equation between spectra and protein standard values. $\mathrm{R}^{2}$, RMSECV and RDP were computed to select the best model. Higher $\mathrm{R}^{2}$ represents that the predictive ability of model is better. RMSECV (cross validation) and RMSEP (external validation) indicate the rate of deviation between predictive value and true value. When they get smaller, models get better. RPD can state the stability of model. A model has a good stability when its RPD $>3$.

In order to eliminate differences, OPUS 6.5 software was used to process spectra obtained by two instruments and to build PLS calibration model. More excellent evaluating indicators and wider modeling wavelength ranges are used to select a better calibration model [4]. Table. 2 shows preprocessing algorithm, wavelength ranges and rank when modeling. 
Table 2. Preprocessing methods, spectral range and rank of the calibration models

\begin{tabular}{cccc}
\hline Instrument & Preprocessing & Regions $\left(\mathrm{cm}^{-1}\right)$ & Rank \\
\hline NIR-WQDS & First derivative & $7500.8-5887.8$ & 5 \\
MPA & First derivative + Vector normalization(SNV) & $12489.5-5446.3$ & 6 \\
\hline
\end{tabular}

\subsection{Results and Analysis}

The relationship between predictive values and standard values in both models is shown in Fig. 3. We can acquire that both models have an excellent goodness of fit, but the linear correlation of MPA is more significant than that of NIR-WDQS.

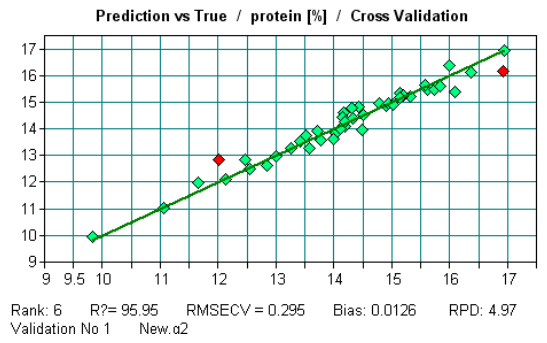

(a)

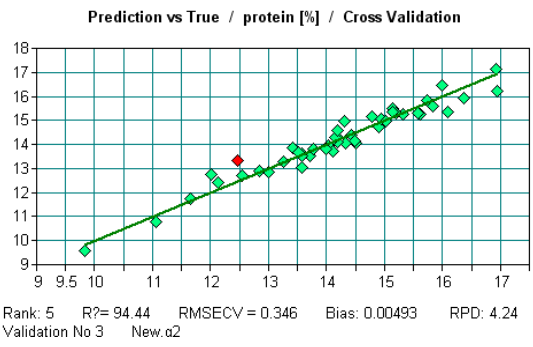

(b)

Fig. 3. The relationship between prediction value and standard value: (a) MPA (b) NIR-WQDS

Evaluating parameters of two models have been shown in Table.3. According to models acquired from NIR-WQDS and MPA, $\mathrm{R}^{2}$ are $94.44 \%$ and $95.95 \%$, RMSECV are $0.3460,0.2950$, RMSEP are 0.2430, 0.2270, and RPD are 4.240 and 4.460, respectively. According to experiment results, forecast performance of NIR-WQDS is good but less excellent than MPA. In the NIR-WQDS, there is a significant linear correlation between standard values and prediction values and the model gets a good predictability and stability [3]. It proves that NIR-WQDS spectroscopy has a good performance with high stability, accuracy and repeatability and it is practical to inspect wheat quality.

Table 3. Optimization results of both models

\begin{tabular}{lllll}
\hline Instrument & $\mathrm{R}^{2}$ & RMSECV & RMESP & RPD \\
\hline NIR-WQDS & $94.44 \%$ & 0.3460 & 0.2430 & 4.240 \\
MPA & $95.95 \%$ & 0.2950 & 0.2270 & 4.970 \\
\hline
\end{tabular}




\section{Conclusion}

NIR wheat quality quick detection system was designed based on grating technology in a spectra range of 900-1700nm. The spectrometer consists of optical system with fixed grating, CCD drive and signal acquisition system, CCD temperature control system, light source control system, sample rotator control system, and computer software system. In this paper, the instrument is proven to be accurate, stable and reliable by analyzing wheat protein content in 46 wheat samples.

The spectra acquired by NIR-WQDS are thorny with spike-waves at extreme ends, which may be caused by noise from slightest vibration of sample rotator, or precision of temperature control system. To improve performance of NIR-WQD, suggestions of the next step are put forward as follows:

(1) Continuing to reduce working temperature of CCD detectors to low dark current.

(2) Adding more processing algorithms and modeling algorithms to perfecting supporting computer software system.

\section{References}

1. Yan, Y.-L.: Foundation and application of near-infrared spectroscopy analysis. China Light Industry Press, Beijing (2005) (in Chinese)

2. Chu, X.-L., Wang, Y.-B., Lu, W.-Z.: Present situation and prospect of near infrared spectrometer in China and abroad. Analytical Instrumentation 4, 1-4 (2007) (in Chinese)

3. Zhang, X.-C., Wu, J.-Z., Xu, Y.: Near infrared spectral analysis technology and its application in modern agriculture. Publishing House of Electronics Industry, Beijing (2012) (in Chinese)

4. Li, J., Zhang, X.-C., Yuan, Y.-W., Zhang, J.-N.: Analysis of soil nutrient content based on near infrared reflectance spectroscopy in Beijing region. Transactions of the CSAE 28(2), 176-179 (2012) (in Chinese)

5. Xiang, X.-Y., Wen, Z.-Y., Long, Z.-C., Hong, M.-J., Liang, Y.-Q., et al.: Research on the near-infrared spectrometer system. Spectroscopy and Spectral Analysis 28(8), 2286-2290 (2009) (in Chinese)

6. Yin, H.-M., Wu, W.-F., Fu, Y., Zhang, Y.-Q., Liu, L.-T.: Designed and test of near infrared detector for grain component based on virtual instrument. Transactions of the Chinese Society for Agricultural Machinery 41(5), 115-119 (2010) (in Chinese)

7. Sun, X.-R., Liu, C.-L., Wu, J.-Z., Suo, S.-Z., Wu, S.-N.: Research of rapid and undamaged assessment of flour quality using near infrared spectroscopy. Journal of Beijing Technology and Business University 29(4), 68-70 (2011) (in Chinese)

8. Zhang, Y.-Q., Zhao, B., Wang, H., Mao, W.-H., Zhang, X.-C.: Technology of near infrared spectroscopy analysis system design based on fixed grating and charge-coupled device. Transactions of the Chinese Society of Agricultural Engineering 25(9), 176-181 (2009) (in Chinese)

9. Jin, H.-L., Xu, C.-H., Xu, Z.-L.: Determination of Protein Content in Wheat Grain by Near Infrared Spectroscopy. Journal of Henan University of Technology (Natural Science Edition) 31(6), 21-24 (2010) (in Chinese) 
10. Balabin, R.M., Safieva, R.Z., Lornakina, E.I.: Wavelet neural network (WNN) approach for calibration model building based on gasoline near infrared (NIR) spectra. Chemometrics and Intelligent Laboratory Systems 93(1), 58-62 (2008)

11. Sundaram, J., Kandala, C.V., Govindarajan, K.N., Subbiah, J.: Sensing of moisture content of in-Shell Peanuts by NIR Reflectance Spectroscopy. Journal of Sensor Technology 2, 1-7 (2012) 